\title{
BMJ Open Healthcare professional and patient codesign and validation of a mechanism for service users to feedback patient safety experiences following a care transfer: a qualitative study
}

\author{
Jason Scott, ${ }^{1}$ Emily Heavey, ${ }^{2}$ Justin Waring, ${ }^{3}$ Diana Jones, ${ }^{4}$ Pamela Dawson ${ }^{4}$
}

To cite: Scott J, Heavey E, Waring $\mathrm{J}$, et al. Healthcare professional and patient codesign and validation of a mechanism for service users to feedback patient safety experiences following a care transfer: a qualitative study. BMJ Open 2016;6:e011222. doi:10.1136/bmjopen-2016011222

\section{- Prepublication history and} additional material is available. To view please visit the journal (http://dx.doi.org/ 10.1136/bmjopen-2016011222).

Received 21 January 2016 Revised 10 June 2016 Accepted 15 June 2016

\section{E CrossMark}

${ }^{1}$ Institute of Health and Society, Newcastle University, Newcastle upon Tyne, UK ${ }^{2}$ Social Policy Research Unit, York University, York, UK ${ }^{3}$ Centre for Health Innovation, Leadership and Learning, Nottingham University, Nottingham, UK

${ }^{4}$ Faculty of Health and Life Sciences, Northumbria University, Newcastle upon Tyne, UK

Correspondence to Dr Jason Scott; jason.scott@ncl.ac.uk

\section{ABSTRACT}

Objective: To develop and validate a mechanism for patients to provide feedback on safety experiences following a care transfer between organisations.

Design: Qualitative study using participatory methods (codesign workshops) and cognitive interviews. Workshop data were analysed concurrently with participants, and cognitive interviews were thematically analysed using a deductive approach based on the developed feedback mechanism.

Participants: Expert patients $(n=5)$ and healthcare professionals $(n=11)$ were recruited purposively to develop the feedback mechanism in 2 workshops. Workshop 1 explored principles underpinning safety feedback mechanisms, and workshop 2 included the practical development of the feedback mechanism. Final design and content of the feedback mechanism (a safety survey) were verified by workshop participants, and cognitive interviews $(n=28)$ were conducted with patients.

Results: Workshop participants identified that safety feedback mechanisms should be patient-centred, short and concise with clear signposting on how to complete, with an option to be anonymous and balanced between positive (safe) and negative (unsafe) experiences. The agreed feedback mechanism consisted of a survey split across 3 stages of the care transfer: departure, journey and arrival. Care across organisational boundaries was recognised as being complex, with healthcare professionals acknowledging the difficulty implementing changes that impact other organisations. Cognitive interview participants agreed the content of the survey was relevant but identified barriers to completion relating to the survey formatting and understanding of a care transfer.

Conclusions: Participatory, codesign principles helped overcome differences in understandings of safety in the complex setting of care transfers when developing a safety survey. Practical barriers to the survey's usability and acceptability to patients were identified, resulting in a modified survey design. Further research is required to determine the usability and acceptability of the survey to patients and healthcare professionals, as well as identifying how

\section{Strengths and limitations of this study}

- This study developed a safety survey using participatory and codesign methods to bring together patient and healthcare professional perspectives.

- Cognitive interviews with 28 patients were used to validate and further refine the survey format and questions.

- Further research is required to pilot the survey to determine whether patients would be willing to be engaged in reporting their experiences of safety following a transfer in care.

- Owing to the nature of organisational care transfers, which potentially include large numbers of organisations, it is unlikely that participants represented all possible types of transfers that patients experience.

- It was not possible to explore further the governance relationships that exist between different organisations responsible for patients' care, which could impact on the implementation of the survey into practice.

governance structures should accommodate patient feedback when relating to multiple health or social care providers.

\section{INTRODUCTION}

Progress in reducing patient harm from adverse incidents in healthcare remains slow. ${ }^{1}$ Involving patients in understanding and commenting on their own safety may help organisations to identify poorly recognised safety issues, improve their learning and safety culture and reduce rates of avoidable harm. ${ }^{2}{ }^{3}$ While advocates of strict safety engineering suggest patients do not have a role to play in their own safety, ${ }^{4}$ it is generally 
argued that, when willing and able, patients should be offered the opportunity to be involved, even though ultimate responsibility for safety rests with care providers. ${ }^{5}$ A recent systematic review identified that patient experience data are positively associated with patient safety and clinical effectiveness. ${ }^{6}$ However, patients often perceive safety differently to clinicians, resulting in a lack of a shared understanding about what it means to feel safe. ${ }^{78}$ In turn, this may impact on the ways in which patients can be involved in their safety.

Patients can be involved in the safety of their care in various ways, ranging from active participation in speaking up and challenging clinicians, ${ }^{9} 10$ through to assessing factors that contribute to safety in hospital settings ${ }^{11} 12$ and reporting safety incidents. ${ }^{13}$ However, it has been identified that formal incident report forms are not an appropriate mechanism for patients to report on their safety because patients were likely to report trivial matters and the process undermined trust in clinicians. ${ }^{14}$ Another way of involving patients is to develop an understanding of, and to coconstruct knowledge about safety. ${ }^{15}$ A recent analysis of patient involvement in safety identified that a conjoint endeavour between patients and clinicians could reduce both parties' anxieties about patient involvement. ${ }^{3}$

Most efforts to involve patients in safety relate to care delivered in a relatively stable secondary care setting, ${ }^{16}{ }^{17}$ in which a single provider is responsible for patient safety. There has been less attention, however, to patient experiences of safety in relation to a transition between organisations, defined as patients moving or being moved from one level of care to another or across different care settings. ${ }^{18}$ The safety implications for care transitions are shown to be complex, resulting from the difficulties of working across organisational boundaries and leading to specific threats to safety and potential for readmission. ${ }^{19}{ }^{20}$ With no one service having overall responsibility for the patient, existing safety systems are negated. In addition, with failures between organisations common, ${ }^{21}$ organisational care transitions arguably increase the risk to patients due to deficits in communication and information transfer which negatively impact on patients' continuity of care. ${ }^{22}$ In Switzerland, it has been reported that poor coordination of care, albeit not necessarily directly related to care transitions, was the most important risk factor about which patients could provide feedback. ${ }^{23}$ As the patient experiences the totality of the transition, there is an opportunity for patients to be involved in the safety of their care by providing a unique perspective on their transition and the continuity of care otherwise unavailable to healthcare professionals. While there are an increasing number of international studies published that have sought to obtain patient perspectives on their transitions between organisations, ${ }^{24} 25$ no known studies have developed, with patients and clinicians, a structured approach to collecting patient feedback on safety experiences in relation to organisational care transitions.
The aim of this study was to fill this knowledge gap by codesigning a mechanism with patients and clinicians for patients to provide feedback on their safety experiences following a transfer between organisations. To achieve this aim, specific objectives were to:

1. Identify principles that should underpin patient feedback on safety experiences following a transfer between organisations;

2. Codesign and construct a feedback mechanism based on these principles and patient perceptions of safety; and

3. Determine the face validity of the survey design with patients who have recently been discharged from hospital.

\section{METHODS}

This study was underpinned by appreciative inquiry (AI), which is a methodology that concentrates on identifying what works well in organisations and attempts to ascertain how these strengths can be built on. ${ }^{20}$ AI is traditionally used as a method of organisational development and is closely aligned to action research, albeit with the emphasis of building on what works well. When used in healthcare, it is often adapted to the requirements of individual projects, ${ }^{21}$ and can even be adapted to underpin specific methods such as appreciative interviews. ${ }^{22}$ The development of the mechanism focused on the 'design' stage of AI, enabling an emphasis on safe rather than unsafe care, which is synonymous with a recent shift in the patient safety movement from what fails occasionally to what succeeds often. ${ }^{23}$

Data were collected across three phases (figure 1), including semistructured interviews with patients that have been published elsewhere ${ }^{8}$ (phase 1). The focus of phase 2 was the development of the feedback mechanism, with phase 3 acting as the validation process of the feedback mechanism. In phase 2 , the primary method of developing the feedback mechanism was via two workshops using participatory and codesign methods, which are receiving increased attention in healthcare for their ability to increase participation and engagement. ${ }^{26}$ The workshops were designed to bring together a wide

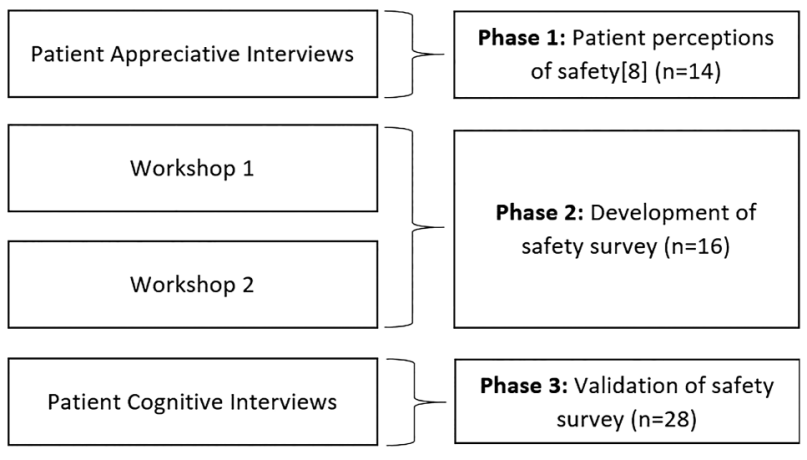

Figure 1 Process of development and validation of the patient feedback mechanism across three phases. 
variety of stakeholders, including patients and healthcare professionals, and afforded the opportunity for different stakeholders to present their unique experiences and perspectives. In phase 3, which was part of a larger feasibility project, ${ }^{27}$ cognitive interviews were used to determine the face validity of the developed feedback mechanism. Patients were involved in choosing the focus on care transitions via the North East Strategic Health Authority's Patient, Carer and Public Engagement Network, who acted as a steering group for the study.

No incentives were provided for participation in any phase of the study.

\section{Phase 1: semistructured interviews}

Semistructured interviews explored the concepts, explanations and terms used by patients when talking about safety in care transfers and how defences, barriers and safeguards can be constructed through the provision of patient-defined safe care. Fourteen participants were interviewed by $\mathrm{JS}$, from three community care teams spanning two National Health Service (NHS) Trusts $(n=7)$, two City Council Resource Centres $(n=3)$, two private nursing and residential care homes $(n=3)$ and via snowball sampling $(\mathrm{n}=1)$ where the participant was not under the care of any organisation at the time of recruitment. A topic guide was used to provide structure to the interviews, with a focus on the types of transfers participants had experienced, whether participants had felt safe during the transfer, what safety meant to participants and what would make participants feel safer in the future. Full details are available in a separate paper. $^{8}$

\section{Phase 2: workshops}

Participants were sampled purposively using criterion sampling $^{28}$ for the two workshops, which were hosted at the Strategic Health Authority and lasted $\sim 2$ hours, to ensure that participants represented different types of organisations involved in the transfer of patients. The patients' voice was provided by five expert patients, identified as such due to their active involvement in either a Patient, Carer and Public Engagement (PCPE) network $(n=3)$, which had also acted as a steering group for the study, or from the Northumbria University Service User Network ( $n=2)$, which consisted of service users who were involved in the education of preregistration and postregistration healthcare professionals.

Eleven healthcare professionals also participated in the workshops. These included NHS community care team nurses $(n=3)$, social care home managers $(n=2)$ and a private nursing home manager $(n=1)$ who were all involved in the identification and recruitment of participants to an earlier phase of the study where perceptions of safety were explored with patients who had recently completed an organisational care transfer. ${ }^{8}$ Additional participants included ambulance service staff $(n=4)$ and a representative of the Strategic Health Authority Patient Safety Team $(n=1)$. Participants were provided with invitation letters and information sheets to explain the purpose of the study, and that participation was voluntary and could be withdrawn at any time.

The first workshop, facilitated by JS and PD, was used to explore the key principles of capturing patient feedback on their experiences of safety. Four questions were posed to the group to ascertain what the feedback mechanism should look like, the format of the feedback mechanism and how the feedback mechanism would fit with current systems. Participants were split into two mixed groups of healthcare professionals and expert patients to discuss answers to the questions. Numerous methods captured discussions to reduce the impact of potential power relationships between healthcare professionals and expert patients, including voice-recordings, flipchart paper, observations and notes from the facilitators and post-it notes.

The second workshop, facilitated by JS and DJ, was structured to have an emphasis on the practical outcome of designing a feedback mechanism, based in part on the results of the first workshop. Components of a Thinking Differently toolkit ${ }^{29}$ were used to encourage creativity among participants when designing the feedback mechanism. Participants were split into two groups and given four Thinking Differently tools; 'fresh eyes', 'reframing by word play', 'pause, notice, observe' and 'random word, picture or object'. ${ }^{29}$ The fundamental basis of this toolkit is that individuals hold schemas, or mental structures of the world, through which thoughts are channelled. The schemas are separated from one another, meaning that it can be difficult to think outside of these mental structures, or to think differently. This in turn inhibits the potential for novel ways of doing something to be introduced into, or alongside, existing systems. Divergent thinking strategies (the Thinking Differently tools) were used in the first half of the workshop (break-out session 1) and participants were encouraged to converge their thinking in the second half of the workshop (break-out session 2; figure 2).

As the workshop data were emergent, it was not possible to plan the data analysis a priori. Instead, for the first workshop, data were analysed inductively based on the different themes and concepts that arose. For the

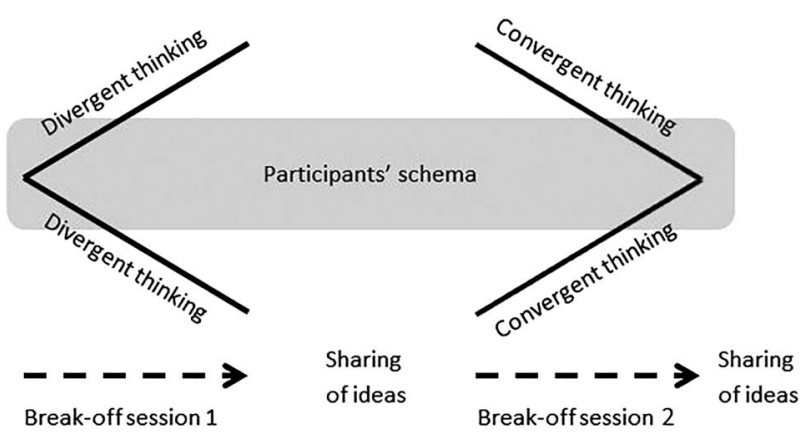

Figure 2 Divergent and convergent thinking strategies in workshop 2 to encourage participants to think outside of their existing feedback mechanism schema. 
second workshop, data analysis was conducted concurrently with participants drawing on each other's ideas and working as individual groups via convergent thinking to assess these shared ideas and bring them into a tangible mechanism for patients to provide feedback on their safety experiences. A final discussion was held with all workshop participants about which parts of each group's chosen feedback mechanism were the strongest. This contributed to a process whereby the participants were involved as coresearchers in data collection and analysis, ${ }^{30}$ occurring in a participatory open forum.

Following the second workshop, a researcher (JS) constructed the survey electronically using the final design agreed by the participants as a template. Additional data that were collected in the second workshop, such as voice recordings and flipcharts, were used postworkshop to ensure that the feedback mechanism had accurately captured what the participants had discussed. On completion, the final design was circulated among all participants for verification that it was an accurate reflection of the discussions and proposed designs. More detail on the construction and content of the survey is provided in the Findings section.

\section{Phase 3: cognitive interviews}

Patients were recruited to cognitive interviews using convenience sampling after completing the safety survey and stating an interest in participating in an interview. Participants completed either the original trifold version of the survey (distribution cycle $1 ; n=20$ ) or an updated bifold version of the survey (distribution cycle 2; $n=8$ ) following discharge from hospital and on arrival at their next destination. Patients deemed unable to give informed consent by their care team or were under the age of 18 were not eligible to participate. Cognitive interviews were conducted by EH and JS with 28 patients (18 male, 10 female) in their place of residence who had completed the safety survey following discharge from hospital. Table 1 provides a summary of the clinical area that the patient was discharged from, distribution cycle recruited from, self-reported transport type and self-reported destination. Participant ages ranged from 53 to 86 (mean=68, SD=10). Cognitive interviews have proved useful in pretesting of survey questions in a healthcare setting, particularly when they may be complex or of a sensitive nature, ${ }^{31}$ as in this study.

Interviewees were invited to describe their thought processes in response to the survey questions, in order to identify any potential misunderstandings or other problems with those questions. We extended this beyond the questions to also ask about other components of the survey, including the introductory text, the description of different sections and the overall structure. Cognitive interviews were audio recorded and transcribed verbatim, then coded and analysed using NVivo qualitative analysis software. Interviews were thematically analysed using a deductive approach based on the structure and

\begin{tabular}{|c|c|c|c|}
\hline Study ID & Cycle & Transport $^{*}$ & Destination* $^{*}$ \\
\hline \multicolumn{4}{|c|}{ Cardiology (n=13) } \\
\hline 980 & 1 & Private car & Hospital \\
\hline 462 & 1 & Private car & Home \\
\hline 2593 & 1 & Ambulance & Hospital \\
\hline 2590 & 1 & Ambulance & Hospital \\
\hline 4679 & 1 & Private car & Hospital \\
\hline 3954 & 1 & Ambulance & Hospital \\
\hline 3319 & 1 & Unknown & Hospital \\
\hline 5945 & 1 & Unknown & Unknown \\
\hline 5583 & 1 & Patient transport & Hospital \\
\hline 4300 & 1 & Private car & Home \\
\hline 6227 & 2 & Private car & Home \\
\hline 6427 & 2 & Private car & Home \\
\hline 11597 & 2 & Taxi & Home \\
\hline \multicolumn{4}{|c|}{ Care of older people $(n=3)$} \\
\hline 104 & 1 & Unknown & Unknown \\
\hline 1189 & 1 & Ambulance & Home \\
\hline 7701 & 2 & Private car & Home \\
\hline \multicolumn{4}{|c|}{ Orthopaedics ( $n=7$ ) } \\
\hline 761 & 1 & Ambulance & Home \\
\hline 1867 & 1 & Private car & Home \\
\hline 2494 & 1 & Ambulance & Home \\
\hline 5853 & 1 & Unknown & Home \\
\hline 6725 & 2 & Private car & Home \\
\hline 9748 & 2 & Private car & Home \\
\hline 11100 & 2 & Walking & Home \\
\hline \multicolumn{4}{|c|}{ Stroke $(n=5)$} \\
\hline 2450 & 1 & Ambulance & Hospital \\
\hline 3445 & 1 & Patient transport & Hospital \\
\hline 3408 & 1 & Private car & Hospital \\
\hline 5767 & 1 & Private car & Home \\
\hline 8182 & 2 & Private car & Home \\
\hline
\end{tabular}

${ }^{*}$ Transport and destination were self-reported. It was not possible to validate or determine the accuracy of this information.

the questions asked in the survey by one researcher $(\mathrm{EH})$, with codes and themes verified by JS, PD and JW.

\section{Findings}

The findings are reported in five sections. The first section summarises the findings of patient perceptions of safety that were published elsewhere. ${ }^{8}$ The next two sections, Principles of patient feedback and Integration with existing systems, represent themes identified in the first workshop that should underpin the development of patient feedback mechanisms applied specifically to capturing patient safety feedback. More specifically, Principles of patient feedback represent the essential design principles of the patient feedback mechanisms, and Integration with existing systems represents the acknowledgement by participants that where multiple organisations are involved in the care of the patient, particularly as patients cross organisational boundaries, feedback needs to be compatible with multiple patient safety and patient experience systems. The last two sections, 
Development of the safety survey and Validation and refinement of the survey report on the development and validation of the survey. These include why participants chose a safety survey as the most appropriate feedback mechanism, how the final design was developed by the participants and cognitive interview findings, including where confusion arose around the question format and the overall survey design.

\section{Patient perceptions of safety}

Semistructured interviews with patients identified aspects of care that had made them feel safe. These included the ways in which staff communicated with patients and responded to the individual needs of the patient, for example, by listening and adjusting the care provided. Interlinked with these themes was that of waiting times, where delays were not communicated to patients and patient requests were not listened to. Patients were also able to identify traditional safety issues, a catch-all term that included medications, falls and healthcare-acquired infections. ${ }^{8}$

\section{Principles of patient feedback}

Participants made recommendations and references to the principles on which the feedback mechanism should be based. There was agreement that the feedback mechanism needed to be short with options to expand on answers, so that service users could report what was of most importance to them. This is highlighted in a conversation during a workshop between a community care team nurse and patient:

From a professional wanting to know what a patient would want, you'd want something that's short but openended... (Community care team nurse)

Yes. (Patient)

So it allows the patient to...discuss one aspect that you felt safe. That's a massive topic but if you had sort of four or five questions like, 'were you happy with that element of care?', 'did you find that was safe?', and that sort of thing. (Community care team nurse)

Yeah, and, 'if not, why not?'. (Patient)

Participants also agreed that a short and concise feedback mechanism would increase response rates. A conversation between a community care team nurse, social care home manager and a patient highlights this agreement, and in doing so they begin to discuss the need for the feedback mechanism to be objective, or unbiased, through the presentation of positive (safe) and negative (unsafe) experiences.

So to capture that [transfers of care are different], would we say that they would want the questionnaire to be sort of short and concise to encourage people to actually do it? (Community care team nurse)
Got to be fairly concise. The longer it is I think the less chance there is of getting involved with it, and especially if you're asking for positive as well as negative feedback or just general commentary. (Social care home manager)

That's a very important point. It shouldn't all be whinging. You need to capture the positives as well. (Patient)

So objective, yeah? (Community care team nurse)

Yeah. (Patient)

This unbiased approach was emphasised by healthcare professionals and patients to emphasise the necessity to be appreciative. In a conversation between a social care home manager and a community care team nurse, the uneven balance of negative rather than positive feedback is discussed. Notably, it was perceived that this imbalance is caused by a lack of recording of positive feedback.

You don't get much feedback unless it's a complaint. (Social care home manager)

But I think, I think a lot of people do get feedback. I just think there's an emphasis on the negative. There's a lot of people, like I'm sure you've probably had a patient, where they feedback that you do a grand job. That never gets captured. (Community care team nurse)

Participants felt that the feedback mechanism should have an option to be anonymous as some service users would want to avoid going through a formal complaints procedure. However, there were concerns over the usability of patient feedback if it could not be traced to a particular incident, thus impacting on potential learning.

The only problem is with it being anonymous is...tracing it back because it's actually more effective when you can look. [...] So you can improve practice generally, but for that specific case you might want to look at it in more detail. (Social care home manager)

\section{Integration with existing systems}

A number of discussion points arose that focused on how the potential feedback mechanism would fit with current feedback mechanisms. First, it was acknowledged that such a system for collecting patient feedback relating to admissions and discharge was required as there was no existing means for patients to provide feedback on this stage of their care, "what we haven't got is just before [service users] get to us, and just after we discharge them" (social care home manager). A paramedic reported that feedback was limited to complaints or compliments, with a gap existing for the routine collection of patient feedback:

We've all got process in place that if there's something we're concerned about we can bring it up. But looking what feedback we get from patients, I know certainly on 
an ambulance point of view, we get no feedback. The only feedback we get is either a complaint coming in or a letter of thanks. (Ambulance service paramedic)

An additional consideration arose in the second workshop, where care home managers from private and social care settings discussed using patient feedback when it relates to care delivered across organisational boundaries. In particular, it was reported and agreed that while patient feedback can be used to change practice, and systems can be changed to incorporate this feedback, they felt there was no opportunity to influence other parts of the health or social care systems. This resulted in a conflicting stance, with healthcare professionals wanting to receive meaningful feedback from patients, but knowing existing organisational structures prohibited being able to respond to this information and change practice. In turn, this had the potential to impact on the utility of any potential feedback mechanism for patients crossing organisational boundaries.

We want instant [patient] feedback to change our systems. [Social care home manager]

And so we can change the system within our environment but we can't change the system anywhere else. (Private nursing care home manager)

\section{Development of the safety survey}

In workshop 2, participants were given four Thinking Differently tools; 'fresh eyes', 'reframing by word play', 'pause, notice, observe' and 'random word, picture or object'. ${ }^{29}$ The use of 'fresh eyes' in particular encouraged participants to explore how non-healthcare organisations approach receiving feedback. These included some of the more traditional feedback mechanisms, such as noticeboards, postcards and questionnaires, and more novel methods, including an aviation-based reporting system, the Royal Society for the Protection of Birds' annual bird watch and supermarket tokens. Table 2 contains a brief description of each of the proposed feedback mechanisms.

Each component of the feedback mechanism was designed by the participants using flipchart paper to draw examples to be discussed. One group decided that the postcard was the best feedback mechanism to take forward and develop due to its simplicity and applicability to a wide variety of settings. This included using a simple scoring system with a three-point scale that incorporated smiley faces: safe (green smiling face), neutral (yellow impassive face) and unsafe (red frowning face).

One side with a smiley face and one side with a.. [unhappy face]. And then straight away you can see. (Private care home manager)

\section{$[\ldots]$}

Table 2 Feedback mechanisms identified by workshop participants for patients to provide feedback on their experiences of safety

\begin{tabular}{|c|c|c|}
\hline Mechanism & Group & Explanation \\
\hline Noticeboard & 1 & $\begin{array}{l}\text { Provided in GP waiting rooms for patients to write comments about their recent } \\
\text { experiences }\end{array}$ \\
\hline Postcard & 1 & $\begin{array}{l}\text { Given to service users during every part of the journey to complete, capturing the wide } \\
\text { range of organisational care transfers }\end{array}$ \\
\hline Post boxes & 1 & $\begin{array}{l}\text { An alternative to the noticeboard which provides privacy for service users and } \\
\text { confidentiality for healthcare professionals }\end{array}$ \\
\hline Thermometer scale & 1 & $\begin{array}{l}\text { Service users are able to place stickers on a large thermometer relating to how safe or } \\
\text { unsafe they felt. Proposed as it would be quick and easy for service users }\end{array}$ \\
\hline Questionnaire & 1 & A simple questionnaire sent to service users post-transfer \\
\hline Aviation Reporting Tool & 2 & $\begin{array}{l}\text { Confidential Human Factors Incident Reporting Programme is used in aviation. } \\
\text { Suggested as an idea as it is confidential and had no blame attributed to the reports }\end{array}$ \\
\hline RSPB Bird Watch & 2 & A method of collecting a lot of data in a systematic way over a short period of time \\
\hline $\begin{array}{l}\text { Gordon Ramsey } \\
\text { approach }\end{array}$ & 2 & $\begin{array}{l}\text { Communication in restaurants by waiters can reduce the impact that long waiting times } \\
\text { have. }\end{array}$ \\
\hline Supermarket tokens & 2 & $\begin{array}{l}\text { System similar to supermarket charity donation tokens. Given to service users on } \\
\text { discharge for them to place in a 'safe' or 'unsafe' box }\end{array}$ \\
\hline Reverse transfer & 2 & $\begin{array}{l}\text { Increase safety by reducing the number of organisational care transfers through } \\
\text { increased care in the community }\end{array}$ \\
\hline Internet questionnaire & 2 & $\begin{array}{l}\text { An automatic email sent to everyone that had gone through an organisational care } \\
\text { transfer }\end{array}$ \\
\hline $\begin{array}{l}\text { Hospital waiting area } \\
\text { information }\end{array}$ & 2 & $\begin{array}{l}\text { Provide information, either in person or via electronic screens regarding length of wait } \\
\text { and delays }\end{array}$ \\
\hline Discharge lounge & 2 & $\begin{array}{l}\text { Place for service users to go prior to a discharge to free up a bed. Somebody could be } \\
\text { there to coordinate transfers, provide information and receive feedback. }\end{array}$ \\
\hline
\end{tabular}


Something simple. I think the most simple ideas are the most effective. (Patient safety team representative)

However, it was also recognised by participants that having an overly simplistic system may result in data that lacked meaning, although participants did not stipulate the minimum or maximum amount of complexity or sensitivity required in order for the data to be meaningful. For example, there was a debate whether a threepoint Likert scale would produce results sensitive enough to identify outliers in safe or unsafe care.

As you were saying where you should have a red, a green, amber, and identifying how happy you were, but the detail this lady's describing would need to be addressed quite intricately. (Ambulance service safeguarding lead)

The other group chose to develop a leaflet-based feedback mechanism, split into three sections directed towards the discharge, transfer and admission of the service user. In particular, their decision to split the transfer into the three stages was summarised by a facilitator (DJ) when feeding back on behalf of the group.

We thought that most journeys, and I like your idea of defining a journey and what service user safety is, have a beginning, and a middle and an end. So, we would like to start with this panel, which is...we've got a day and a date...place of departure, so where did you depart from? (Facilitator, DJ)

This three-stage structure was used in the final design, although transfer was changed to journey after the workshop, following feedback from one participant during verification of the design. Table 3 provides an overview of the survey structure and questions.

I'm never happy with transfer because people...some people, particularly the public, would automatically think you're talking about wheels, as opposed to the journey. (Email correspondence, community care team nurse)

\section{Validation and refinement of the survey through cognitive interviews}

Twenty participants provided feedback on an original trifold version of the survey. There was some diversity of opinion on the appropriateness of the paper format and the three-face design. While some participants suggested that an online or telephone survey might be easier to complete, there was a general consensus that varying access to computers, as well as time and cost restraints, meant that a paper version was more appropriate for most people. Patient 1867 summarised:

I would quite happily fill it in on an App, but [people] who are not computer literate would just back away from that. I think paperwork is probably the best way that would cover every age group. (Patient 1867)
Most participants found the three-point scale with smiley faces easy to use and understand. The statement from patient 4300 makes this point, as well as reinforcing the workshop participants' preference for the survey to be concise:

Smiley faces and sad faces and things like that, you know red faces, it looked simple, it was easy, it caught your eye. It wasn't too wordy cos I think there's nothing worse than wordy surveys where you get half way through and you think, 'You know what, I can't be bothered'. (Patient 4300)

However, it should be noted that some participants expressed a preference for 'yes/no/maybe' style questions, with one suggesting that asking whether a patient felt safe, neutral or unsafe was confusing and even 'loaded' (patient 3954). Another participant suggested that three faces were not enough, and that there should be five in varying shades. Despite this diversity, there was general agreement that the paper survey with the three faces tick-box system was easy to use.

It was reported that two aspects of the survey design caused difficulties for many participants; the division into three stages of the care transfer (departure, journey, arrival) and the way in which the questions were asked. For the stages of the transfer, patients were unclear about which departure, journey and arrival they were being asked. Some interpreted the questions in the 'journey' section to be relating to their journey to hospital rather than from hospital or thought they were being asked 'to give an average' assessment of the two journeys (P1189); others thought that 'arrival' referred to their initial arrival on the ward, rather than at their next destination.

The format of that is not right. It needs drastically changing, I think you should keep 'your departure from' that needs to be explained really, from where? (Patient 3954)

Second, some participants did not make the distinction between these three stages at all, instead answering questions in the three separate sections in relation to the entirety of the care transfer; these participants saw the three separate sections as merely repeating the same questions, without distinguishing between different transfer stages. For example, patient 5853, when asked how they had interpreted a question relating to 'arrival', stated:

[The answer given does not relate to] when I was at home, I was talking probably, I thought this was probably an overall of those. (Interviewer): 'Your Arrival' as a summary of everything else? (Patient 5853): Yeah.

On the basis of these findings, the survey was restructured into a two-page leaflet. The front and back pages provided additional information about the survey, and the middle two pages contained the survey questions 
Table 3 Structure and question format of the safety survey following initial development

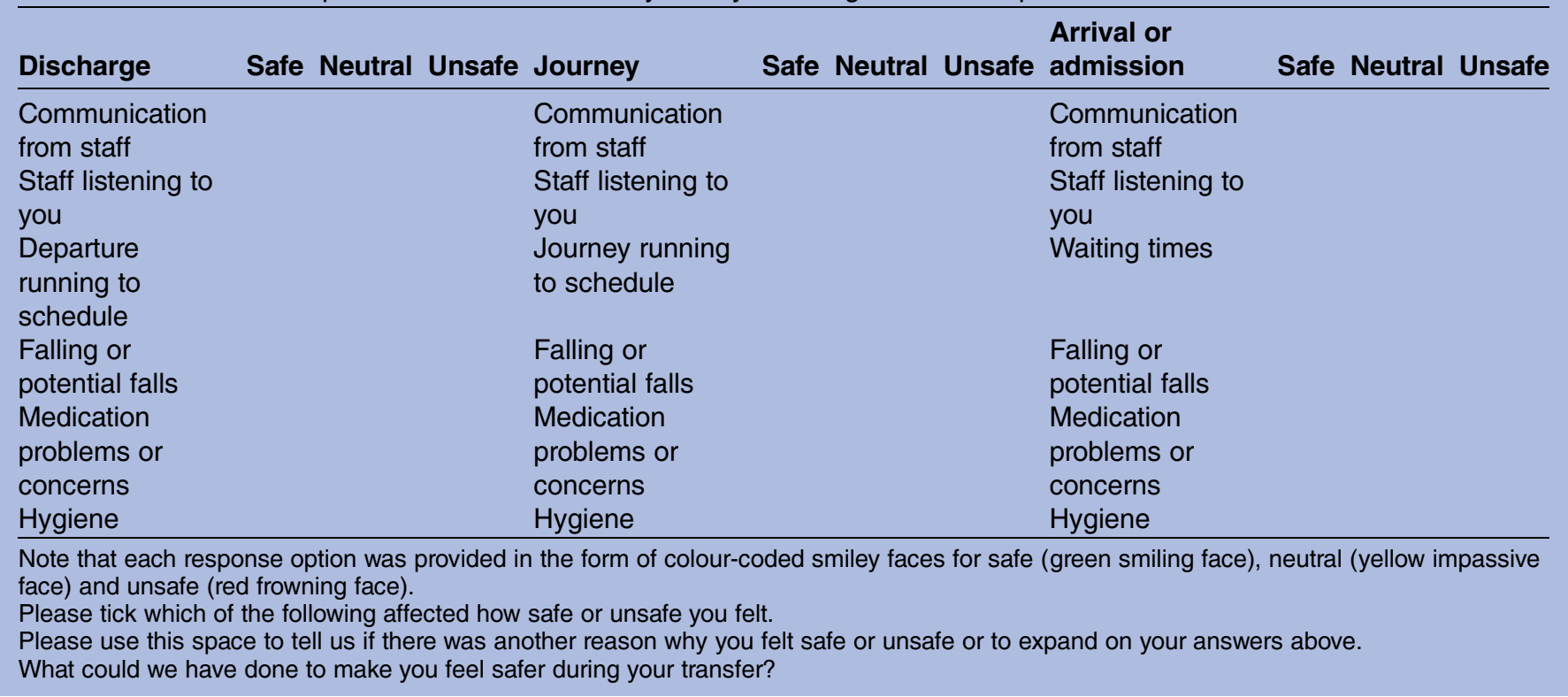

(box 1). The survey still asked questions about each of the three stages of the transfer (departure, journey and arrival); however, this was asked within each question. An additional explanation of the stages of the transfer was provided with increased clarity over which transfer was being referred to, and the survey questions were expanded to be more specific about what was being asked (see online supplementary material for the wording). Space for free-text comments was provided next to each question. Cognitive interviews with eight additional patients using the modified version of the survey suggested that the changes had resolved the original issues around question clarity and the type of transfer that was being asked about. Participants suggested that some sections of the survey were not of relevance to them, which was either due to patients feeling safe, or because parts of their transfer did not involve healthcare staff, such as when transported by private car.

[The only difficulty completing it was] knowing what on earth to put sometimes, because I kept thinking, 'I don't think, I don't think that applies.'[...] I couldn't decide whether I was putting the right thing sometimes, because I didn't feel unsafe and y'know, everything was kind of looked after okay. (Patient 6227)

Similar to the original trifold design, patients also reported that they considered the survey to be capturing their experiences of safety across their entire episode of care, rather than an individual transfer. For example, patient 6725 reflected, "this felt as though it was reflecting on my three day stay in hospital", and patient 8182 provided a similar reflection. This suggests that the description of the stages of the transfer was not sufficient in explaining to patients that the survey was focusing only on the transfer, and not their entire episode of care, and future iterations would require this distinction to be explicit.

I wasn't sure that it was [the transfer] that they were asking the question for, or that it was a general safety survey of the whole experience of going to hospital, being a patient. (Patient 8182)

\section{DISCUSSION}

A number of systematic reviews consider how patients can provide feedback on their safety; ${ }^{14} 16 \quad 17$ however, these focus on adverse events, typically within discrete care settings such as secondary care, rather than experiences of safety in the context of care transitions. Furthermore, there are relatively few studies reporting on the development of these feedback mechanisms. One notable study has reported on the development of a patient reporting tool, though again this is specific to secondary care settings. ${ }^{11-13}$ Our study developed a mechanism for patients to provide feedback on their safety experiences following a transfer between organisations through a process of codesign. The transfer between organisations was chosen as it is a time in the patient's episode of care that is acknowledged to be particularly high in risk, ${ }^{21} 32$ and when mistakes are likely to occur. $^{33}$

The developed safety survey aims to capture patient experiences of safety, based on patients' definitions of what it is that makes them feel safe during a care transition. $^{8}$ This is a notable shift from some existing approaches to involving patients in reporting patient safety incidents, which have had limited success. ${ }^{14}$ There has been a limited amount of work attempting to reconcile the differing perceptions of safety between clinicians 
Box 1 Question format of the safety survey following cognitive interviews. Response options are provided in square brackets. Note that each response option was provided in the form of colour-coded smiley faces for safe (green smiling face), neutral (yellow impassive face) and unsafe (red frowning face)

How safe did communication from staff make you feel? For example, giving you clear and timely information or being polite.

- On your departure (safe/neutral/unsafe);

- During your journey (safe/neutral/unsafe);

- On arrival at your next destination (safe/neutral/unsafe).

How safe did you feel with regard to staff listening to you and responding to your individual needs?

- On your departure (safe/neutral/unsafe);

- During your journey (safe/neutral/unsafe);

- On arrival at your next destination (safe/neutral/unsafe).

Did you experience any delays? (yes/no)

- If yes, where was your longest delay? (departure/journey/ arrival)

- How did this make you feel? (safe/neutral/unsafe)

How safe did you feel about the possibility of falling? For example, if you felt confident that you would not fall or if you were concerned that you might.

- On your departure (safe/neutral/unsafe);

- During your journey (safe/neutral/unsafe);

- On arrival at your next destination (safe/neutral/unsafe).

How safe did you feel about your medication? For example, receiving the correct medication, understanding the medication you were taking or delays in receiving your medication.

- On your departure (safe/neutral/unsafe);

- During your journey (safe/neutral/unsafe);

- On arrival at your next destination (safe/neutral/unsafe).

How safe did you feel about hygiene and cleanliness? For example, if staff washed their hands and if the surroundings were clean.

- On your departure (safe/neutral/unsafe);

- During your journey (safe/neutral/unsafe);

- On arrival at your next destination (safe/neutral/unsafe).

Overall, how safe did you feel throughout the whole transfer including the departure, journey and arrival? (safe/neutral/unsafe)

and patients that result in a lack of a shared understanding about what it means to feel safe, ${ }^{78}$ but the use of codesign approaches in developing feedback mechanisms can go some way to bringing together the different perceptions, particularly as it has been identified that patient experiences can be linked to clinical safety. ${ }^{6}$

By bringing together patients and healthcare professionals in tailored workshops within this study, we were able to identify principles that should underpin the feedback mechanism, including that it should be patientcentred, short, concise with clear signposting on how to complete it, optionally anonymous and be objective with a focus on positive (safe) and negative (unsafe) care.

Within the principles of feedback mechanisms, the provision of patient-centred care refers to the location of the patient within their care. For a feedback mechanism to be patient-centred, this in turn requires the opportunity for patients to be involved and to play an active role, thereby placing their experience of care at the forefront. The length and structure of the feedback mechanism, in being short and concise with clear signposting on how to complete it, is already a feature of patient experience surveys and the benefits of brevity include increased response rates and greater acceptability and usability among patients. ${ }^{34}$

Giving patients the option to provide anonymous feedback is particularly important when considering and discussing safety. Existing evidence suggests that patients have concerns, whether founded or not, that challenging healthcare professionals can impact on the care received and engender feelings of suspicion and mistrust, ${ }^{35}$ and the concept of providing anonymous feedback was enshrined in participants' comments and the final feedback mechanism designed in this study. That both patients and healthcare professionals identified the need for feedback to be balanced between positive and negative experiences demonstrates that both groups were aware of criticisms of existing feedback mechanisms that focus on negative experiences alone, such as the use of complaints. The paradox of measuring safety by its absence was acknowledged early in the patient safety movement, ${ }^{36}$ but this is now being reflected in proactive approaches to safety, ${ }^{37}$ and the findings of this study suggest that the same principle should be applied to patient feedback mechanisms. The principles of being patient-centred, short, concise with clear signposting on how to complete the feedback mechanism, optionally anonymous and objective with a focus on positive and negative care can be applied by others who are interested in developing feedback mechanisms for patients to provide feedback on their experiences of safety, and the generic nature of the principles can be applied to settings other than organisational care transfers.

Finally, the complexity associated with care being received across organisational boundaries was identified by participants and is recognised elsewhere in the literature. ${ }^{20}$ In particular, healthcare professionals in this study acknowledged that they would be unable to implement change that impacts on or requires the input of other service providers as a result of patient feedback. This was a significant outcome, and an important consideration for future research that aims to involve the patient in their safety across organisational boundaries. Agreement between, or integration of, services may be necessary in order to promote organisational learning and change service delivery in response to patient feedback.

\section{USE OF CODESIGN METHODS}

We built on the principles that should underpin a feedback mechanism by using participatory and codesign methods in the development of the survey, which are receiving increased attention in healthcare for their ability to increase participation and engagement, ${ }^{26}$ and we used the Thinking Differently methodology ${ }^{29}$ to provide a means by which to break out of existing schemas to encourage innovation. 
Furthermore, codesigning a feedback mechanism ensures that it meets the requirements of different groups of users; in the case of this study, patients who are required to understand and complete the questions, and healthcare professionals who are required to collect and learn from the feedback provided. Codesign was particularly important given the differences that exist in patients' and healthcare professionals' understandings of safety, and provided an opportunity for shared learning. Despite these benefits of using codesign, we did encounter challenges associated with the approach, including personal agendas and dominant voices. Prior to the first workshop, we developed inclusive strategies such as post-it notes and flipchart paper that would enable both patient and healthcare professional participants to have their voice heard, even if it was not audible. ${ }^{38}$ The issue of personal agendas among participants, where they would attempt to overly influence the direction of discussion, was a greater challenge. In a systematic review of the impact on patient involvement on research, personal experience stories that dominated discussions were identified to be a challenge ${ }^{39}$ In order to resolve this, we used the Thinking Differently toolkit in workshop 2 to provide focus for all participants by directing thoughts and discussions to situations equally familiar to all, thus reducing the available space in which individuals could dominate discussions.

\section{LIMITATIONS}

This study developed a safety survey using participatory and codesign methods, including the identification of underlying principles. While the survey was codesigned by healthcare professionals and patients, including cognitive interviews to validate and further refine the survey, further research is required to pilot the developed feedback mechanism to determine whether patients would be willing to be engaged in reporting their experiences of safety following a transfer in care. Furthermore, the participants involved in the development of the survey were recruited to represent a wide variety of health and social care services and patients. Owing to the nature of organisational care transfers, it is unlikely that they represented all possible types of transfers that patients experience. It was also not possible to explore further the governance relationships that exist between organisations, regardless of representation in this study, which could impact on the implementation of the survey into practice. Finally, the self-reported transport and destination of the cognitive interview participants was not directly explored, and so it was not possible to validate or determine the accuracy of this information.

\section{FUTURE RESEARCH}

The use of participatory and codesign principles helped to overcome differences in the understanding of safety, to develop a feedback mechanism for patients to provide feedback on their experiences of safety relating to a care transfer. Additional research is required before the survey is ready to be used in practice, including piloting in further clinical areas in order to determine its usability and acceptability to patients and healthcare professionals. Patient cognitive interviews indicated confusion between whether patients were being asked to provide feedback solely on their care transfer or their whole episode of care, indicating that it may be difficult to solicit feedback on experiences of care relating to one aspect of an episode of care. Further research is required to explore this, which could include determining whether asking patients about safety experiences is likely to increase awareness of patient safety, and whether patient experiences of safety can lead to quality improvement in the complex area of care transfers.

Twitter Follow Pamela Dawson at @ProfPamDawson, Jason Scott at @DrJasonScott and Justin Waring at @dr_waring

Acknowledgements The authors would like to thank all participants for their invaluable contribution to the study, the Safer Care North East Patient, Carer and Public Engagement group and the PRoSOCT advisory group for their support and guidance, and the National Health Service (NHS) Trusts involved in the study for their ongoing support. They also thank The Health Foundation (an independent charity committed to bringing about better health and healthcare for people in the UK), Northumbria University and the former North East Strategic Health Authority, in particular the Patient Safety Action Team and the Safer Care North East programme, for funding the studies that contributed to this paper. The research team acknowledge the support of the National Institute for Health Research, through the Comprehensive Clinical Research Network.

Contributors Phases 1 and 2: JS, PD and DJ designed the phases, collected, analysed and interpreted the data. Phase 3: JS, JW and PD designed the phase. JS and EH collected, analysed and interpreted the data, with input from JW and PD. All authors contributed to the drafting of the manuscript.

Funding This research was funded by the North East Strategic Health Authority, Northumbria University and The Health Foundation (grant number 7204).

Competing interests None declared.

Ethics approval Approval for phases 1 and 2 was provided by Northumbria University School of Health, Community and Education Studies Ethics Committee, Sunderland Research Ethics Committee (reference: 09:H0904/57) and R\&D departments at each of the included NHS sites. Approval for phase 3 was provided by Yorkshire \& The Humber-Leeds West Research Ethics Committee (reference: 13/YH/0372) and R\&D departments at each of the included NHS sites.

Provenance and peer review Not commissioned; externally peer reviewed.

Data sharing statement No additional data are available.

Open Access This is an Open Access article distributed in accordance with the Creative Commons Attribution Non Commercial (CC BY-NC 4.0) license, which permits others to distribute, remix, adapt, build upon this work noncommercially, and license their derivative works on different terms, provided the original work is properly cited and the use is non-commercial. See: http:// creativecommons.org/licenses/by-nc/4.0/

\section{REFERENCES}

1. Shojania KG, Thomas EJ. Trends in adverse events over time: why are we not improving? BMJ Qual Saf 2013;22:273-7.

2. Schwappach DL. Review: engaging patients as vigilant partners in safety: a systematic review. Med Care Res Rev 2010;67:119-48.

3. Sutton E, Eborall H, Martin G. Patient involvement in patient safety: current experiences, insights from the wider literature, promising opportunities? Public Manag Rev 2015;17:72-89. 
4. Lyons M. Should patients have a role in patient safety? A safety engineering view. Qual Saf Health Care 2007;16:140-2.

5. Davis RE, Jacklin R, Sevdalis N, et al. Patient involvement in patient safety: what factors influence patient participation and engagement? Health Expect 2007;10:259-67.

6. Doyle C, Lennox L, Bell D. A systematic review of evidence on the links between patient experience and clinical safety and effectiveness. BMJ Open 2013;3:e001570.

7. Weissman JS, Schneider EC, Weingart SN, et al. Comparing patient-reported hospital adverse events with medical record review: do patients know something that hospitals do not? Ann Intern Med 2008;149:100-8.

8. Scott J, Dawson P, Jones D. Do older patients' perceptions of safety highlight barriers that could make their care safer during organisational care transfers? BMJ Qual Saf 2012;21:112-17.

9. Rainey $\mathrm{H}$, Ehrich $\mathrm{K}$, Mackintosh $\mathrm{N}$, et al. The role of patients and their relatives in 'speaking up' about their own safety-a qualitative study of acute illness. Health Expect 2015;18:392-405.

10. Davis RE, Sevdalis N, Vincent CA. Patient involvement in patient safety: how willing are patients to participate? BMJ Qual Saf 2011:20:108-14.

11. McEachan RR, Lawton RJ, O'Hara JK, et al. Developing a reliable and valid patient measure of safety in hospitals (PMOS): a validation study. BMJ Qual Saf 2014;23:565-73.

12. Giles SJ, Lawton RJ, Din I, et al. Developing a patient measure of safety (PMOS). BMJ Qual Saf 2013;22:554-62.

13. Ward J, McEachan R, Lawton R, et al. Patient involvement in patient safety: protocol for developing an intervention using patient reports of organisational safety and patient incident reporting. BMC Health Serv Res 2011;11:130.

14. Doherty C, Stavropoulou C, Dickinson L. Patients' willingness to complete written incident report forms in one UK tertiary cancer hospital. Clin Risk 2015;21:77-82.

15. Rhodes $\mathrm{P}$, McDonald R, Campbell S, et al. Sensemaking and the co-production of safety: a qualitative study of primary medical care patients. Sociol Health IIIn 2016;38:270-85.

16. King A, Daniels J, Lim J, et al. Time to listen: a review of methods to solicit patient reports of adverse events. Qual Saf Health Care 2010;19:148-57.

17. Massó Guijarro P, Aranaz Andrés JM, Mira JJ, et al. Adverse events in hospitals: the patient's point of view. Qual Saf Health Care 2010;19:144-7.

18. Holland DE, Harris MR. Discharge planning, transitional care, coordination of care, and continuity of care: clarifying concepts and terms from the Hospital perspective. Home Health Care Serv $Q$ 2007;26:3-19.

19. Coleman EA, Smith JD, Frank JC, et al. Preparing patients and caregivers to participate in care delivered across settings: the care transitions intervention. J Am Geriatr Soc 2004;52:1817-25.

20. Waring J, Marshall F, Bishop S. Understanding the occupational and organizational boundaries to safe hospital discharge. J Health Serv Res Policy 2015;20(Suppl 1):35-44.

21. Jeffs L, Lyons RF, Merkley J, et al. Clinicians' views on improving inter-organizational care transitions. BMC Health Serv Res 2013;13:289.
22. Kripalani S, LeFevre F, Phillips CO, et al. Deficits in communication and information transfer between hospital-based and primary care physicians: implications for patient safety and continuity of care. JAMA 2007:297:831-41.

23. Schwappach DL. Frequency of and predictors for patient-reported medical and medication errors in Switzerland. Swiss Med Wkly 2011;141:w13262.

24. Flink $\mathrm{M}$, Öhlén $\mathrm{G}$, Hansagi $\mathrm{H}$, et al. Beliefs and experiences can influence patient participation in handover between primary and secondary care-a qualitative study of patient perspectives. BMJ Qual Saf 2012;21(Suppl 1):i76-83.

25. Flink M, Hesselink G, Pijnenborg L, et al. The key actor: a qualitative study of patient participation in the handover process in Europe. BMJ Qual Saf 2012;21(Suppl 1):i89-96.

26. Bessant $J$, Maher $L$. Developing radical service innovations in healthcare-the role of design methods. Int $J$ Innov Manag 2009;13:555-68.

27. Scott J, Waring J, Heavey E, et al. Patient Reporting of Safety experiences in Organisational Care Transfers (PRoSOCT): a feasibility study of a patient reporting tool as a proactive approach to identifying latent conditions within healthcare systems. BMJ Open 2014;4:e005416.

28. Palys T. Purposive sampling. In: Given LM, ed. The Sage encyclopedia of qualitative research methods. Lose Angeles: Sage, 2008:697-8.

29. Maher L, Plsek P, Garrett S, et al. Thinking Differently: concepts, tools and methods to unblock thinking in health care. Coventry: NHS Institute for Innovation and Improvement, 2007.

30. Baum F, MacDougall C, Smith D. Participatory action research J Epidemiol Community Health 2006;60:854-7.

31. Drennan J. Cognitive interviewing: verbal data in the design and pretesting of questionnaires. J Adv Nurs 2003;42: 57-63.

32. Cook RI, Render M, Woods DD. Gaps in the continuity of care and progress on patient safety. BMJ 2000;320:791-4.

33. Fuji KT, Abbott AA, Norris JF. Exploring care transitions from patient caregiver, and health-care provider perspectives. Clin Nurs Res 2013;22:258-74.

34. The Health Foundation. Evidence scan: measuring patient experience. London: The Health Foundation, 2013.

35. Hrisos S, Thomson R. Seeing it from both sides: do approaches to involving patients in improving their safety risk damaging the trust between patients and healthcare professionals? An interview study. PLOS ONE 2013;8:e80759.

36. Reason J. Safety paradoxes and safety culture. Inj Control Saf Promot 2000;7:3-14.

37. Hollnagel E. Thought paper: proactive approaches to safety management. London: The Health Foundation, 2012.

38. Elberse JE, Caron-Flinterman JF, Broerse JEW. Patient-expert partnerships in research: how to stimulate inclusion of patient perspectives. Health Expect 2011;14:225-39.

39. Brett J, Staniszewska S, Mockford C, et al. Mapping the impact of patient and public involvement on health and social care research: a systematic review. Health Expect 2014;17:637-50. 